\title{
On the calm b-differentiability of projector onto circular cone and its applications
}

\author{
Vo Duc Thinh*

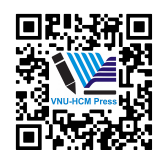 \\ Use your smartphone to scan this \\ QR code and download this article
}

Dong Thap University

Correspondence

Vo Duc Thinh, Dong Thap University

Email: vdthinh@dthu.edu.vn

History

- Received: 2020-07-30

- Accepted: 2020-09-06

- Published: 2020-10-10

DOI : 10.32508/stdj.v23i4.2426

\section{Check for updates}

\section{Copyright}

(c) VNU-HCM Press. This is an openaccess article distributed under the terms of the Creative Commons Attribution 4.0 International license.

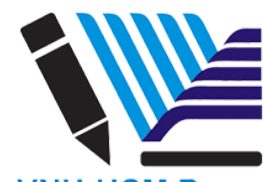

VNU-HCM Press

\begin{abstract}
In this paper, we study a concept on the calm B-differentiability, a new kind of generalized differentiabilities for a given vector function introduced by Ye and Zhou in 2017, of the projector onto the circular cone. Then, we discuss its applications in mathematical programming problems with circular cone complementarity constraints. Here, this problem can be considered to be a generalization of mathematical programming problems with second-order cone complementarity constraints, and thus it includes a large class of mathematical models in optimization theory. Consequently, the obtained results for this problem are generalized, and then corresponding results for some special mathematical problems can be implied from them directly. For more detailed information, we will first prove the calmly B-differentiable property of the projector onto the circular cone. This result is not easy to be shown by simply resorting to those of the projection operator onto the second-order cone. By virtue of exploiting variational techniques, we next establish the exact formula for the regular (Fréchet) normal cone (this concept was proposed by Kruger and Mordukhovich in 1980) to the circular cone complementarity set. Note that this set can be considered to be a generalization of the second-order cone complementarity set. In finally, the exact formula for the regular (Fréchet) normal cone to the circular cone complementarity set would be useful for us to study first-order necessary optimality conditions for mathematical programming problems with circular cone complementarity constraints. Our obtained results in the paper are new, and they are generalized to some existing ones in the literature.
\end{abstract}

Key words: calmly B-differentiable, circular cone, complementarity set, Fréchet normal cone, optimality condition

\section{INTRODUCTION}

The second-order cone programming (SOCP) problem plays an important role in the optimization theory and has attracted much attention from mathematicians, see, e.g., ${ }^{1-7}$. We refer the reader to ${ }^{1,2,4-7}$ and the references therein for some remarkable results on optimality conditions and stability analysis of (SOCP).

Inspired by the second-order cone, many researchers have investigated optimization and complementarity problems where their constraints are involved in second-order cones. It is called the second-order cone complementarity problem (SOCCP), which includes a large class of optimization problems such as quadratically constrained problems $\left(\mathrm{see}^{8}\right)$, the second-order cone programming, and nonlinear complementarity problem $\left(\mathrm{see}^{9}\right)$. In particular, recent attention is paid to the second-order cone complementarity set.

Let us now mention some existing results concerning this set. In ${ }^{10}$, Liang et al. provided formulations for Fréchet normal cone to the second-order cone complementarity set. Unfortunately, the obtained results were shown to be inexact in ${ }^{11}$. In that paper, Ye and Zhou gave exact formulas for the proximal/regular (Fréchet)/limiting normal cone to the second-order cone complementarity set by using the projection operator onto second-order cones and the generalized differentiability called the calm B-differentiability. Some first-order optimality conditions for mathematical programs with second-order cone complementarity constraints were obtained in ${ }^{12}$ and sufficient conditions for error bound property of second-order cone complementarity problems were established in ${ }^{13}$. To obtain these results, the authors used the symmetric and self-dual property of the second-order cone.

Recently, generalizations of second-order cones and second-order cone complementarity sets have been examined by many authors ${ }^{5,14-22}$. For example, authors in ${ }^{14,19-22}$ considered circular cones, which are generalizations of second-order cones and are, in general, nonsymmetric and non-self-dual cones. The generalized differentiability of the projection operator 
onto the circular cone was provided in ${ }^{14,22}$. Moreover, the differentiability and calmness of vectorvalued functions associated with the circular cone were also studied in ${ }^{19,23}$. In particular, authors in ${ }^{21}$ showed that the results of the projection operator onto a circular cone could not be shown by simply resorting to the results of the projection operator onto the second-order cone, and hence, it is necessary to study the results of circular cone directly.

To the best of our knowledge, there is no result on the calmly B-differentiable property concerning the circular cone and its extension. In this paper, inspired by ${ }^{11,13,22}$, we first study in Section 3, the calm B-differentiability of the circular cone. We then provide in Section 4 the formula for the Fréchet normal cone to a circular cone complementarity set, which can be considered as a generalization of the secondorder cone complementarity set. This formula would be useful for us to study optimality conditions for mathematical programming problems with circular cone complementarity constraints.

\section{PRELIMINARIES}

Throughout the paper, if not otherwise specified, $f(t)=o(t) \quad(f(t)=O(t))$ means $\frac{f(t)}{|t|} \rightarrow 0$ (resp., $\frac{f(t)}{|t|}$ is uniformly bounded) as $t \rightarrow 0$, and $(f(x))_{+}:=$ $\max \{f(x), 0\}$, and $(f(x))_{-}:=\min \{f(x), 0\} \cdot B_{r}(x)$ stands for the closed ball centered at $x \in \mathbb{R}^{n}$ with radius $r>0$. Given $x, y \in \mathbb{R}^{n}, x^{\mathrm{T}} y$ stands for the scalar product of $x$ and $y$. For $x:=\left(x_{0}, x_{r}\right) \in \mathbb{R} \times \mathbb{R}^{n-1}$, we use the following notation

$$
\begin{aligned}
& x^{\perp}:=\left\{y \in \mathbb{R}^{n} \mid x^{\mathrm{T}} y=0\right\} \text { and } \\
& \widetilde{\mathrm{x}}_{\mathrm{r}}:=\left\{\begin{array}{l}
\frac{x_{r}}{\left\|x_{r}\right\|} \text { if } x_{r} \neq 0, \\
\text { any unit vector } \mathrm{e} \in \mathbb{R}^{\mathrm{n}-1} \text { if otherwise. }
\end{array}\right.
\end{aligned}
$$

Let $\mathrm{C} \subset \mathbb{R}^{n}$ be a nonempty subset, $\mathrm{cl} C$ denotes its closure. The polar cone $\mathrm{C}^{\circ}$ and the dual cone $C^{\star}$ of $C$ are

$$
\begin{aligned}
& C^{\circ}:=\left\{y \in \mathbb{R}^{n} \mid y^{\top} x \leq 0, \forall x \in C\right\} \text { and } \\
& C^{\star}:=\left\{y \in \mathbb{R}^{n} \mid y^{\top} x \geq 0, \forall x \in C\right\}
\end{aligned}
$$

respectively.

The Fréchet normal cone to $C$ at $\mathrm{x} \in \mathrm{cl} C$ are defined respectively by, see ${ }^{24}$,

$$
\begin{aligned}
& \widehat{N}_{C}(x):= \\
& \left\{x^{*} \in \mathbb{R}^{n}\left\langle x^{*}, x^{\prime}-x\right\rangle \leq o\left(\left\|x^{\prime}-x\right\|\right), \forall x^{\prime} \in C\right\} .
\end{aligned}
$$

Lemma $2.1\left({ }^{24}\right.$, Theorem 1.14) Let $\mathrm{D}=\{\mathrm{x} \mid \mathrm{h}(\mathrm{x}) \in C\}$ and let $\nabla \mathrm{h}(\mathrm{x})$ be surjective. Then

$$
\widehat{N}_{D}(x)=\nabla h(x)^{T} \widehat{N}_{C}(x) .
$$

Let $f: \mathbb{R}^{n} \rightarrow(-\infty, \infty]$ and $\bar{x} \in \mathbb{R}^{n}$ such that $f(\bar{x})$ is finite. The Fréchet subdifferential of $\mathrm{f}$ at $\bar{x}$ is defined by, see [ ${ }^{24}$, pages 89 and 90$]$,

$$
\begin{aligned}
& \widehat{\partial} f(\bar{x}):=\left\{x^{*} \in \mathbb{R}^{n}\right\} \mid \\
& \limsup \sin _{x \rightarrow \bar{x}} \frac{\left\langle x^{*}, x-\bar{x}\right\rangle-f(x)+f(\bar{x})}{\|x-\bar{x}\|} \leq 0
\end{aligned}
$$

The indicator function of a set $C \subset \mathbb{R}^{n}$ is denoted by

$$
\delta_{C}(x):=\left\{\begin{array}{l}
0 \text { if } x \notin C, \\
\infty \text { otherwise }
\end{array}\right.
$$

It is known from [ ${ }^{25}$, Proposition 1.18] that $\widehat{\partial} \delta_{C}(x)=$ $\widehat{N}_{C}(x)$

for any $x \in C$.

Let $\mathrm{F}: \mathbb{R}^{n} \Rightarrow \mathbb{R}^{m}$ be a set-valued mapping, the domain and the graph of $F$ are

$$
\begin{aligned}
& \operatorname{dom} F:=\left\{x \in \mathbb{R}^{n} \mid F(x) \neq \varnothing\right\}, \\
& \operatorname{gph} F:=\left\{(x, y) \in \mathbb{R}^{n} \times \mathbb{R}^{m} \mid y \in F(x)\right\} .
\end{aligned}
$$

The Fréchet coderivative of $F$ at $(x, y) \in \mathrm{gph} F$ are respectively defined by, see [ ${ }^{24}$, Definition 1.32], for each $\mathrm{y}^{*} \in \mathbb{R}^{m}$,

$$
\begin{aligned}
& \widehat{D}^{*} F(x, y)\left(y^{*}\right) \\
& :=\left\{x^{*} \in \mathbb{R}^{n} \mid\left(x^{*},-y^{*}\right) \in \widehat{N}_{\mathrm{gph} F}(x, y)\right\} .
\end{aligned}
$$

When $F(x)$ is single-valued, $y$ can be omitted in the above notations. Moreover, if $F$ is continuously differentiable, then for all $\mathrm{y}^{*} \in \mathbb{R}^{m}$, we get

$$
\widehat{D} * F(x)(y *)=\{\nabla F(x) * y *\} .
$$

The derivative in the directionh $\in \mathbb{R}^{n}$ of $F$ at $x$ is defined by

$$
F^{\prime}(x ; y):=\lim _{t \rightarrow 0^{+}} \frac{F(x+t h)-F(x)}{t} .
$$

The circular cone is defined (cf. ${ }^{14,19-23}$ ) by

$$
\begin{aligned}
& \mathscr{K}_{\theta}:= \\
& \left\{x=\left(x_{0}, x_{r}\right) \in \mathbb{R} \times \mathbb{R}^{m}\left|x_{0} \tan \theta \geq \| x_{r}\right| \mid\right\}
\end{aligned}
$$

with angle $\theta \in\left(0, \frac{\pi}{2}\right)$. When $\theta=\frac{\pi}{4}$, it reduces to the second-order cone defined by $\mathscr{K}_{\theta}:=$ $\left\{x=\left(x_{0}, x_{r}\right) \in \mathbb{R} \times \mathbb{R}^{m}\left|x_{0} \geq \| x_{r}\right| \mid\right\}$. In this case, the set

$$
\Omega:=\left\{(\mathrm{x}, \mathrm{y}) \mid \mathrm{x} \in \mathscr{K}, \mathrm{y} \in \mathscr{K}, \mathrm{x}^{\mathrm{T}} \mathrm{y}=0\right\},
$$

is called the second-order cone complementarity set. If $\theta \neq \frac{\pi}{4}$ then $\mathscr{K}_{\theta}$ is a nonsymmetric and non-self-dual 
cone. The boundary and the interior of $\mathscr{K}_{\theta}$ are given respectively by

$$
\begin{aligned}
& \text { bd } \mathscr{K}_{\theta}:= \\
& \left\{x=\left(x_{0}, x_{r}\right) \in \mathbb{R} \times \mathbb{R}^{m} \mid x_{0} \tan \theta \geq\left\|x_{r}\right\|\right\}, \\
& \text { int } \mathscr{K}_{\theta}:=\mathscr{K}_{\theta} \backslash\left(\text { bd } \mathscr{K}_{\theta}\right) .
\end{aligned}
$$

The positive dual cone and the polar cone of $\mathscr{K}_{\theta}$ are defined respectively by, see [ ${ }^{20}$, Theorem 2.1$]$,

$$
\begin{aligned}
& \mathscr{K}_{\theta}:=\left\{y=\left(y_{0}, y_{r}\right) \in \mathbb{R} \times \mathbb{R}^{m}\left|y_{0} \cot \theta \geq \| y_{r}\right| \mid\right\}, \\
& \mathscr{K}_{\theta}^{*}:=-\mathscr{K}_{\theta}^{*}= \\
& \left\{y=\left(-y_{0},-y_{r}\right) \in \mathbb{R} \times \mathbb{R}^{m} \mid y_{0} \cot \theta \geq\left\|y_{r}\right\|\right\} .
\end{aligned}
$$

A relation between the boundary of $\mathscr{K}_{\theta}$ and that of $\mathscr{K}_{\theta}^{*}$ is established as follows.

Proposition 2.2 Let $x \in b d \mathscr{K}_{\theta} \backslash\{0\} \quad$ and $y \in b d \mathscr{K}_{\theta}^{*} \backslash\{0\}$. Then, $y^{T} x=0$

if and only if $x=\kappa\left(y_{0} \cot ^{2} \theta,-y_{r}\right)$ with $k=$ $\frac{x_{0}}{y_{0}} \tan ^{2} \theta$ (equivalently, $y=k\left(x_{0} \tan ^{2} \theta,-x_{r}\right)$ with $k=$ $\frac{y_{0}}{x_{0}} \cot ^{2} \theta$.).

Proof. Let $x \in \operatorname{bd} \mathscr{K}_{\theta} \backslash\{0\}$ and $y \in \operatorname{bd} \mathscr{K}_{\theta}^{*} \backslash\{0\}$.

"If": Suppose that there exists $\kappa \in \mathbb{R}_{++}:=(0, \infty)$ with $x=\kappa\left(y_{0} \cot ^{2} \theta,-y_{r}\right)$, then $y^{T} x=0$.

"Only if": Let $y^{T} x=0$, then we get $x_{0} \tan \theta=\left\|x_{r}\right\|$ $y_{0} \tan \left(\frac{\pi}{2}-\theta\right)=\left\|y_{r}\right\|$ and $\mathrm{x}_{0} \mathrm{y}_{0}+\mathrm{y}_{\mathrm{r}}^{\mathrm{T}} \mathrm{x}_{\mathrm{r}}=0$. Thus, one has

$$
\begin{aligned}
& y_{r}^{T} x_{r}=-x_{0} y_{0}=\left(\left\|x_{r}\right\| \cot \theta\right) \times \\
& \left(\left\|y_{r}\right\| \cot \left(\frac{\pi}{2}-\theta\right)\right)=-\left\|x_{r}\right\| \cdot\left\|y_{r}\right\|,
\end{aligned}
$$

which implies the existence of $\kappa \in \mathbb{R}_{++}$such that $x_{r}=$ $-\kappa y_{r}$. Consequently, we obtain

$$
x_{0} \tan \theta=\kappa y_{0} \tan \left(\frac{\pi}{2}-\theta\right),
$$

i.e., $\mathrm{x}_{0} \tan ^{2} \theta=\kappa y_{0}$. Hence, $x=\kappa\left(y_{0} \cot ^{2} \theta,-y_{r}\right)$ with $\kappa=\frac{x_{0}}{y_{0}} \tan ^{2} \theta$, and the proof is completed.

We recall that for any given $x:=\left(x_{0}, x_{r}\right) \in \mathbb{R} \times \mathbb{R}^{\mathrm{m}}$, it can be decomposed by (see $\left[{ }^{20}\right.$, Theorem 3.1])

$$
x=\lambda_{1}(x) u_{x}^{1}+\lambda_{2}(x) u_{x}^{2},
$$

where the spectral values $\lambda_{1}(x), \lambda_{2}(x)$ and the spectral vectors $u_{x}^{1}, u_{x}^{2}$ are defined respectively by

$$
\begin{gathered}
\lambda_{1}(x):=x_{0}-\left\|x_{r}\right\| \cot \theta, \\
\lambda_{2}(x):=x_{0}+\left\|x_{r}\right\| \tan \theta, \\
u_{x}^{1}:=\frac{1}{1+\cot ^{2} \theta}\left[\begin{array}{cc}
1 & 0 \\
0 & \cot \theta
\end{array}\right]\left[\begin{array}{c}
1 \\
-\widetilde{x}_{r}
\end{array}\right], \\
u_{x}^{2}:=\frac{1}{1+\tan ^{2} \theta}\left[\begin{array}{cc}
1 & 0 \\
0 & \tan \theta
\end{array}\right]\left[\begin{array}{c}
1 \\
\widetilde{x}_{r}
\end{array}\right] .
\end{gathered}
$$

The metric projection of $x$ onto $\mathscr{K}_{\theta}$, denoted by $\Pi_{\mathscr{K}_{\theta}}(\mathrm{x})$, is defined as follows

$$
\begin{aligned}
& \Pi_{\mathscr{K}_{\theta}}(\mathrm{x}):=\arg \min _{\mathrm{z} \in \mathscr{K}_{\theta}}\|\mathrm{x}-\mathrm{z}\| \\
& =\left\{\mathrm{z} \in \mathscr{K}_{\theta} \mid\|\mathrm{x}-\mathrm{z}\| \leq\|\mathrm{x}-\mathrm{u}\|, \forall \mathrm{u} \in \mathscr{K}_{\theta}\right\} .
\end{aligned}
$$

From $^{22}$ and the convexity of $\mathscr{K}_{\theta}$, we get that $\Pi_{\mathscr{K}_{\theta}}(\mathrm{x})$ is a single-valued set and

$$
\Pi_{\mathscr{K}_{\theta}}(\mathrm{x})=\left(\lambda_{1}(\mathrm{x})_{+}\right) \mathrm{u}_{\mathrm{x}}^{1}+\left(\lambda_{2}(\mathrm{x})_{+}\right) \mathrm{u}_{\mathrm{x}}^{2} .
$$

Moreover, $\left[{ }^{26}\right.$, Proposition 2] states that, for all $\mathrm{x} \in$ $\mathbb{R}^{\mathrm{m}+1}$,

$$
\begin{aligned}
& \mathrm{x}=\Pi_{\mathscr{K}_{\theta}}(\mathrm{x})+\Pi_{\mathscr{K}_{\theta}^{\circ}}(\mathrm{x}) \\
& \text { and }\left\langle\Pi_{\mathscr{K}_{\theta}}(\mathrm{x}), \Pi_{\mathscr{K}_{\theta}^{\circ}}(\mathrm{x})\right\rangle=0 .
\end{aligned}
$$

Since $\Pi\left(\mathscr{K}_{\theta}\right)(\mathrm{x}), \Pi\left(\mathscr{K}_{\theta}^{\circ}\right)(\mathrm{x})$, one gets

$$
\Pi_{\mathscr{K}_{\theta}}(\mathrm{x})=-\Pi_{\mathscr{K}_{\theta}^{\circ}}(-\mathrm{x})
$$

Let us define the circular cone complementarity set as

$$
\Gamma:=\left\{(x, y) \mid x \in \mathscr{K}_{\theta}, y \in \mathscr{K}_{\theta}^{*}, \mathrm{x}^{\mathrm{T}} \mathrm{y}=0\right\},
$$

which is a generalized type of (2.2). Given $(x, y) \in \Gamma$ and an arbitrary $u \in \mathscr{K}_{\theta}$, it holds that

$$
\begin{aligned}
& \|(x-y)-u\|^{2}-\|(x-y)-x\|^{2} \\
& =\|(x-u)-y\|^{2}-\|y\|^{2} \\
& =\|x-u\|^{2}-2\langle x-u, y\rangle \\
& =\|x-u\|^{2}-2\langle x, y\rangle+2\langle u, y\rangle \geq 0,
\end{aligned}
$$

which means that $x=\Pi_{\mathscr{K}_{\theta}}(x-y)$. Similarly, we get that $y \in \Pi_{\mathscr{K}_{\theta}^{*}}(y-x)$.

The above observation allows us to obtain a relation between the complementarity set $\Gamma$, and the projection onto $\mathscr{K}_{\theta}$ as follows.

Proposition 2.3 Let $\Gamma$ be as in (2.4). Then, we get

$$
\begin{aligned}
& {[(x, y) \in \Gamma] \Longleftrightarrow\left[x \in \Pi \mathscr{K}_{\theta}(x-y)\right]} \\
& \Longleftrightarrow\left[y \in \Pi_{\mathscr{K}_{\theta}^{*}}(y-x)\right] \Longleftrightarrow\left[-y \in \Pi_{\mathscr{K}_{\theta}^{\circ}}(x-y)\right] .
\end{aligned}
$$

By Proposition 2.3, $\Gamma$ can be expressed by

$$
\Gamma=\left\{(x, y) \mid(x-y, x) \in \operatorname{gph} \Pi_{\mathscr{K}_{\theta}}\right\} .
$$

Let $f: \mathbb{R}^{m+1} \times \mathbb{R}^{m+1} \rightarrow \mathbb{R}^{m+1} \times \mathbb{R}^{m+1}$ be defined by $f(x, y):=(x-y, x)$ for all $(x, y) \in \mathbb{R}^{m+1} \times \mathbb{R}^{m+1}$, we can check that $f$ is continuously differentiable and

$$
\nabla f(x, y)=\left[\begin{array}{cc}
I_{m+1} & -I_{m+1} \\
I_{m+1} & 0
\end{array}\right],
$$

where $I_{m+1}$ is the unit matrix of the degree $m+1$, has full rank. It follows from [ ${ }^{27}$, Exercise 6.7] that

$$
\begin{aligned}
& \widehat{N}_{\Gamma}(x, y)= \\
& \left\{\nabla f(x, y)^{*}\left(x^{*}, y^{*}\right) \mid\left(x^{*}, y^{*}\right) \in \widehat{N}_{g p h \Pi_{\mathscr{K}_{\theta}}}(f(x, y))\right\} \\
= & \left\{x^{*}+y^{*},-x^{*}\right) \mid\left(x^{*}, y^{*}\right) \in \widehat{N}_{\left.\left.g p h \Pi_{\mathscr{K}_{\theta}}\right)(x-y, x)\right\}} \\
= & \left\{(u, v) \mid-v \in \widehat{D}^{*} \Pi_{\mathscr{K}_{\theta}}(x-y)(-u-v)\right\} .
\end{aligned}
$$


From the above discussion, one obtains the following result, which plays an important role in computing the Fréchet normal cone to complementarity set.

Proposition 2.4 Let $\Gamma$ be as in (2.4) and $(\mathrm{x}, \mathrm{y}) \in \Gamma$. Then, we get

$$
\begin{aligned}
& \widehat{N}_{\Gamma}(x, y) \\
& =\left\{(u, v) \mid-v \in \widehat{D}^{*} \Pi_{\mathscr{K}_{\theta}}(x-y)(-u-v)\right\} .
\end{aligned}
$$

\section{CALM B-DIFFERENTIABILITY OF THE PROJECT MAPPING ONTO A CIRCULAR CONE}

In this section, we first show that the projection operator $\Pi_{\mathscr{K}_{\theta}}$ is calmly B-differentiable at any $x \in \mathbb{R}^{m+1}$. Then, we provide a characterization for a proximal normal vector of $\Gamma$.

Definition 3.1 ( $\left.{ }^{11}\right)$ The function $F: \mathbb{R}^{n} \rightarrow \mathbb{R}^{m}$ is called calmly B-differentiable at $x$ if, for all h sufficiently close to 0 , we get

$$
F(x+h)-F(x)-F^{\prime}(x ; h)=O\left(\|h\|^{2}\right) .
$$

Theorem 3.2 The projection mapping $\Pi_{\mathscr{K}_{\theta}}$ is calmly B-differentiable for any $x \in \mathbb{R}^{m+1}$.

Proof.Given an arbitrary $x \in \mathbb{R}^{m+1}$, it is enough to show that, for $h$ sufficiently close to 0 ,

$$
\begin{gathered}
\Pi_{\mathscr{K}_{\theta}}(x+h)-\Pi_{\mathscr{K}_{\theta}}(x)-\Pi_{\mathscr{K}_{\theta}}^{\prime}(x ; h) \\
=0=O\left(\|h\|^{2}\right) .
\end{gathered}
$$

We consider the following cases.

Case 1: $x \in \operatorname{int} \mathscr{K}_{\theta}$. Then, we have $\Pi_{\mathscr{K}_{\theta}}(x)=$ $x, \Pi_{\mathscr{K}_{\theta}}(x+h)=x+h$. Moreover, it follows from the definition of the directional derivative that $\Pi_{\mathscr{K}_{\theta}}^{\prime}(x ; h)=h$. So, (3.1) is fulfilled.

Case 2: $x \in-\operatorname{int} \mathscr{K}^{*}$. We get, in this case that $\Pi_{\mathscr{K}_{\theta}}(x)=0, \Pi_{\mathscr{K}_{\theta}}(x+h)=0$. On the other hand, by the definition of $\Pi_{\mathscr{K}_{\theta}}^{\prime}(x ; h)$, one has $\Pi_{\mathscr{K}_{\theta}}^{\prime}(x ; h)=0$, so (3.1) holds.

Case 3: $x \in b d \mathscr{K}_{\theta} \backslash\{0\}$. It implies that $\lambda_{1}(x)=0$ and $\lambda_{2}(x)>0$. By (2.3) and Lemma 3.2(b) in ${ }^{22}$, we have

$$
\begin{aligned}
& \Pi_{\mathscr{K}_{\theta}}(x)=x, \Pi_{\mathscr{K}_{\theta}}^{\prime}(x ; h) \\
& =h-\left(1+\cot ^{2} \theta\right)\left(\left(u_{1}^{x}\right)^{\mathrm{T}} h\right) \_u_{1}^{x},
\end{aligned}
$$

$$
\begin{aligned}
& \Pi_{\mathscr{K}_{\theta}}(x+h)=\left(\left(x_{0}+h_{0}-\left\|x_{r}+h_{r}\right\| \cot \theta\right)_{+}\right) \\
& \times \frac{1}{1+\cot ^{2} \theta}\left[\begin{array}{c}
1 \\
\frac{\left(-x_{r}-h_{r}\right) \cot \theta}{\left\|x_{r}+h_{r}\right\|}
\end{array}\right] \\
& +\left(\left(x_{0}+h_{0}+\left\|x_{r}+h_{r}\right\| \tan \theta\right)_{+}\right) \\
& \times \frac{1}{1+\tan ^{2} \theta}\left[\begin{array}{c}
1 \\
\frac{\left(x_{r}+h_{r}\right) \tan \theta}{\left\|x_{r}+h_{r}\right\|}
\end{array}\right] .
\end{aligned}
$$

Let $\widetilde{\Pi}^{h}=\left(\widetilde{\Pi}_{0}^{h}, \widetilde{\Pi}_{r}^{h}\right) \in \mathbb{R}^{m+1}$ be defined by

$$
\widetilde{\Pi}^{h}=\Pi_{\mathscr{K}_{\theta}}(x+h)-\Pi_{\mathscr{K}_{\theta}}(x)-\Pi_{\mathscr{K}_{\theta}}^{\prime}(x ; h)
$$

we now show computations for $\widetilde{\Pi}_{0}^{h}$ and $\widetilde{\Pi}_{r}^{h}$. By (3.2) and (3.3), $\widetilde{\Pi}_{0}^{h}$ is given by Figure 1 .

By the expression of $\left\|x_{r}\right\|$, for all $h_{r}$ sufficiently close to 0 , we get $\left\|x_{r}+h_{r}\right\|=\left\|x_{r}\right\|+\left\langle\widetilde{x}_{r}, h_{r}\right\rangle+O\left(\left\|h_{r}\right\|^{2}\right)$, which implies that

$$
\begin{aligned}
& x_{0}+h_{0}-\left\|x_{r}+h_{r}\right\| \cot \theta \\
& =h_{0}-\left\langle\widetilde{x}_{r}, h_{r}\right\rangle \cot \theta+O\left(\left\|h_{r}\right\|^{2}\right) .
\end{aligned}
$$

It follows from (3.4), (3.5), and the Lipschitz property of the function $(\cdot)_{\text {_ }}$ with modulus 1 that

$$
\begin{aligned}
& \mid-\left(x_{0}+h_{0}-\left\|x_{r}+h_{r}\right\| \cot \theta\right)_{-} \\
& +\left(h_{0}-\left\langle\widetilde{x}_{r}, h_{r}\right\rangle \cot \theta\right)_{-} \mid \leq O\left(\left\|h_{-} r\right\|^{2}\right) .
\end{aligned}
$$

On the other hand, we also get equation 3.7 in Figure 2 Using the Taylor expression of the function $\frac{x}{\|x\|}$, one has

$$
\begin{aligned}
& \frac{x_{r}+h_{r}}{\left\|x_{r}+h_{r}\right\|} \\
& =\widetilde{x}_{r}+\frac{1}{\|x\|}\left(\widetilde{x}_{r}, \widetilde{x}_{r}^{\mathrm{T}}\right) h_{r}+O\left(\left\|h_{r}\right\|^{2}\right) .
\end{aligned}
$$

Thus, (3.7) is given by Figure 3

Moreover, from (3.5) and (3.6), we obtain

$$
\begin{aligned}
& \widetilde{\Pi}_{r}^{h}=\frac{\left(\widetilde{x}_{r}, \widetilde{x}_{r}^{\mathrm{T}}\right) h_{r}+O\left(\left\|h_{r}\right\|^{2}\right)}{\left\|x_{r}\right\|(\cot \theta+\tan \theta)} \times \\
& \left(\left(h_{0}-\left\langle x_{r}, h_{r}\right\rangle \cot \theta+O\left(\left\|h_{r}\right\|^{2}\right)\right)_{-}\right) \\
& \left.+\frac{\widetilde{x}_{r}}{\cot \theta+\tan \theta}\left(\left\|h_{r}\right\|^{2}\right)\right) .
\end{aligned}
$$

It is necessary to show that $\left\|\widetilde{\Pi}_{r}^{h}\right\| \leq O\left(\|h\|^{2}\right)$. Indeed, it follows from (3.10) that

$$
\begin{aligned}
& \left\|\widetilde{\Pi}_{r}^{h}\right\| \leq \frac{\left\|\left(\widetilde{x}_{r}, \widetilde{x}_{r}^{\mathrm{T}}\right) h_{r}+O\left(\left\|h_{r}\right\|^{2}\right)\right\|}{\left\|x_{r}\right\|(\cot \theta+\tan \theta)} \times \\
& \left(\|h\| \sqrt{1+\cot ^{2} \theta}+O\left(\|h\|^{2}\right)\right) \\
& +O\left(\|h\|^{2}\right)=O\left(\|h\|^{2}\right) .
\end{aligned}
$$

Combining to (3.6), we get $\left\|\widetilde{\Pi}^{h}\right\|=O\left(\|h\|^{2}\right)$, which means that (3.1) holds.

Case 4: $x \in-b d \mathscr{K}_{\theta}^{*} \backslash\{0\}$. It is obvious that $\Pi_{\mathscr{K}_{\theta}}(x)=$ 0 . Then, for each $h \in \mathbb{R}^{m+1}$ sufficiently close to 0 , we have $\lambda_{1}(x)<0, \lambda_{2}(x)=0$ and $\lambda_{1}(x+h)=\left(x_{0}+\right.$ $\left.h_{0}\right)-\left\|x_{r}+h_{r}\right\| \cot \theta<0$.. It follows from (2.3) and Lemma 3.2 in $^{22}$ that

$\Pi_{\mathscr{K}_{\theta}}(x+h)=\left(\left(x_{0}+h_{0}+\tan \theta\left\|x_{r}+h_{r}\right\|\right)_{+}\right) u_{x+h}^{2}$ $\Pi_{\mathscr{K}_{\theta}}^{\prime}(x ; h)=\left(1+\tan ^{2} \theta\right)\left(\left\langle u_{x}^{2}, h\right\rangle\right)+u_{x}^{2}$,

where

$$
\begin{gathered}
u_{x}^{2}=\frac{1}{1+\tan ^{2} \theta}\left[\begin{array}{c}
1 \\
\widetilde{x}_{r} \tan \theta
\end{array}\right], \\
u_{x+h}^{2}=\frac{1}{1+\tan ^{2} \theta}\left[\begin{array}{c}
1 \\
\frac{x_{r}+h_{r}}{\left\|x_{r}+h_{r}\right\|} \tan \theta
\end{array}\right] \\
=u_{x}^{2}+\frac{1}{1+\tan ^{2} \theta}\left[\begin{array}{c}
1 \\
\frac{1}{\left\|x_{r}\right\|}\left(\widetilde{x}_{r} \tilde{x}_{r}^{\mathrm{T}}\right) h_{r}+O\left(\left\|h_{r}\right\|^{2}\right)
\end{array}\right]
\end{gathered}
$$




$$
\begin{gathered}
\frac{1}{\left\|x_{r}+h_{r}\right\|(\cot \theta+\tan \theta)}\left[\tan \theta\left\|x_{r}+h_{r}\right\|\left(\left(x_{0}+h_{0}-\left\|x_{r}+h_{r}\right\| \cot \theta\right)_{+}\right)\right. \\
\left.+\cot \theta\left\|x_{r}+h_{r}\right\|\left(x_{0}+h_{0}+\left\|x_{r}+h_{r}\right\| \tan \theta\right)_{+}\right]-x_{0}-h_{0}+\left(\left(u_{x}^{1}\right)^{\top} h\right)_{-} \\
=\frac{1}{\left\|x_{r}+h_{r}\right\|(\cot \theta+\tan \theta)}\left[\operatorname { t a n } \theta \| x _ { r } + h _ { r } \| \left(\left(x_{0}+h_{0}-\left\|x_{r}+h_{r}\right\| \cot \theta\right)\right.\right. \\
\left.\left.-\left(x_{0}+h_{0}-\left\|x_{r}+h_{r}\right\| \cot \theta\right)_{-}\right)+\cot \theta\left\|x_{r}+h_{r}\right\|\left(x_{0}+h_{0}+\left\|x_{r}+h_{r}\right\| \tan \theta\right)_{+}\right] \\
-x_{0}-h_{0}+\frac{1}{1+\cot ^{2} \theta}\left(h_{0}-\left\langle\tilde{x}_{r}, h_{r}\right\rangle \cot \theta\right)_{-} \\
=-\frac{\left(x_{0}+h_{0}-\left\|x_{r}+h_{r}\right\| \cot \theta\right)_{-}}{1+\cot ^{2} \theta}+\frac{1}{1+\cot ^{2} \theta}\left(h_{0}-\left\langle\tilde{x}_{r}, h_{r}\right\rangle \cot \theta\right)_{-} .
\end{gathered}
$$

Figure 1: Equation 3.4

$$
\begin{aligned}
& \widetilde{\Pi}_{r}^{h}=\frac{x_{r}+h_{r}}{\left\|x_{r}+h_{r}\right\|(\cot \theta+\tan \theta)}\left[\left(x_{0}+h_{0}+\left\|x_{r}+h_{r}\right\| \tan \theta\right)-\left(x_{0}+h_{0}-\| x_{r}+h_{r}\right.\right. \\
& \quad-x_{r}-h_{r}-\frac{\tilde{x}_{r}}{\cot \theta+\tan \theta}\left(h_{0}-\left\langle\tilde{x}_{r} \cot \theta, h_{r}\right\rangle\right)_{-} \\
& =\frac{x_{r}+h_{r}}{\left\|x_{r}+h_{r}\right\|(\cot \theta+\tan \theta)}\left(x_{0}+h_{0}-\left\|x_{r}+h_{r}\right\| \cot \theta\right)_{-}-\frac{\tilde{x}_{r}}{\cot \theta+\tan \theta}\left(h_{0}-\left\langle\tilde{x}_{r} \cot \theta, h_{r}\right\rangle\right)_{-} .
\end{aligned}
$$

Figure 2: Equation 3.7

$$
\begin{gathered}
\widetilde{\Pi}_{r}^{h=} \frac{\tilde{x}_{r}+\left(\tilde{x}_{r} \tilde{x}_{r}^{\top}\right) h_{r}+o\left(\left\|h_{r}\right\|^{2}\right)}{\cot \theta+\tan \theta}\left(\left(x_{0}+h_{0}-\left\|x_{r}+h_{r}\right\| \cot \theta\right)_{-}\right)-\frac{\tilde{x}_{r}}{\cot \theta+\tan \theta}\left(h_{0}-\left\langle\tilde{x}_{r} \cot \theta, h_{r}\right\rangle\right)_{-} \\
\quad=\frac{\tilde{x}_{r}}{\cot \theta+\tan \theta}\left(x_{0}+h_{0}-\left\|x_{r}+h_{r}\right\| \cot \theta\right)_{-\left(h_{0}-\left(\tilde{x}_{r} \cot \theta, h_{r}\right)_{-}\right.} \\
\quad+\frac{\left(\tilde{x}_{r} \tilde{x}_{r}^{\top}\right) h_{r}+o\left(\left\|h_{r}\right\|^{2}\right)}{\left\|x_{r}\right\|(\cot \theta+\tan \theta)}\left(x_{0}+h_{0}-\left\|x_{r}+h_{r}\right\| \cot \theta\right)_{-}
\end{gathered}
$$

Figure 3: Equation 3.9

Thus, one gets

$$
\begin{gathered}
\widetilde{\Pi}^{h}=\left(x_{0}+h_{0}+\tan \theta\left\|x_{r}+h_{r}\right\|\right)_{+} u_{x+h}^{2} \\
-\left(1+\tan ^{2} \theta\right)\left(\left\langle u_{x}^{2}, h\right\rangle\right)+u_{x}^{2} \\
=\left(h_{0}+\left\langle\widetilde{x}_{r} \tan \theta, h_{r}\right\rangle+O\left(\left\|h_{r}\right\|^{2}\right)\right)_{+} \\
\left(u_{x}^{2}+\frac{1}{1+\tan ^{2} \theta}\left[\begin{array}{c}
1 \\
0 \\
\left\|x_{r}\right\|
\end{array}\left(\widetilde{x}_{r} \widetilde{x}_{r}^{\mathrm{T}}\right) h_{r}+O\left(\left\|h_{r}\right\|^{2}\right)\right]\right) \\
-\left(h_{0}+\left\langle\widetilde{x}_{r} \tan \theta, h_{r}\right\rangle\right)+u_{x}^{2}
\end{gathered}
$$

with $\widetilde{\Pi}^{h}:=\Pi_{\mathscr{K}_{\theta}}(x+h)-\Pi_{\mathscr{K}_{\theta}}(x)-\Pi_{\mathscr{K}_{\theta}}^{\prime}(x ; h)$. Since the function $(\cdot)_{+}$is Lipschitz with modulus 1 , from
(3.11), we obtain

$$
\begin{aligned}
&\left\|\widetilde{\Pi}^{h}\right\| \leq O\left(\left\|h_{r}\right\|^{2}\right)+\left\|u_{x}^{2}\right\| \\
&+\|\left(h_{0}+\left\langle\widetilde{x}_{r} \tan \theta\right\rangle+O\left(\left\|h_{r}\right\|^{2}\right)\right)_{+} \\
&\left(\frac{1}{1+\tan ^{2} \theta}\right)\left[\begin{array}{c}
1 \\
\frac{1}{\left\|x_{r}\right\|}\left(\widetilde{x}_{r} \widetilde{x}_{r}^{\mathrm{T}}\right) h_{r}+O\left(\left\|h_{r}\right\|^{2}\right)
\end{array}\right] \| \\
& \leq O\left(\left\|h_{r}\right\|^{2}\right)+\left(\|h\| \sqrt{1+\tan ^{2} \theta}+O\left(\left\|h_{r}\right\|^{2}\right)\right. \\
& \times\left(\frac{1}{1+\tan ^{2} \theta} \sqrt{\left(\frac{1}{\left\|x_{r}\right\|^{2}} \widetilde{x}_{r}, h_{r}\right)^{2}+O\left(\left\|h_{r}\right\|^{4}\right)}\right) \\
&=O\left(\|h\|^{2}\right) .
\end{aligned}
$$

Note that the last inequality holds by $\left\|h_{r}\right\| \leq\|h\|$. Consequently, (3.1) is implied.

Case 5: $\mathrm{x}=0$. Then, for all $h \in \mathbb{R}^{m+1}$, we get

$$
\begin{aligned}
& \lambda_{1}(x)=\lambda_{2}(x)=0 \text { and } \\
& \Pi_{\mathscr{K}_{\theta}}(x)=0, \Pi_{\mathscr{K}_{\theta}}(x+h)=\Pi_{\mathscr{K}_{\theta}}(h), \\
& \Pi_{\mathscr{K}_{\theta}}^{\prime}(x)=\Pi_{\mathscr{K}_{\theta}}(h) .
\end{aligned}
$$


Thus, one has

$$
\left\|\Pi_{\mathscr{K}_{\theta}}(x+h)-\Pi_{\mathscr{K}_{\theta}}(x)-\Pi_{\mathscr{K}_{\theta}}^{\prime}(x ; h)\right\|=0,
$$

which means that (3.1) holds.

Case 6: $\quad x \in \mathbb{R}^{m+1} \backslash\left(\mathscr{K}_{\theta} \cup\left(-\mathscr{K}_{\theta}^{*}\right)\right)$. Since $\mathbb{R}^{m+1} \backslash\left(\mathscr{K}_{\theta} \cup\left(-\mathscr{K}_{\theta}^{*}\right)\right)$ is open, for $\mathrm{h}$ sufficiently close to 0 , one has $x+h \in \mathbb{R}^{m+1} \backslash\left(\mathscr{K}_{\theta} \cup\left(-\mathscr{K}_{\theta}^{*}\right)\right)$. Moreover, we can check that $\lambda_{1}(x)<0, \lambda_{1}(x+h)<0$ and $\lambda_{2}(x)>0, \lambda_{2}(x+h)>0$. Thus, it follows from (2.3) and $\left[{ }^{22}\right.$, Lemma 3.2(a) and (3.6)] that

$$
\begin{aligned}
& \Pi_{\mathscr{K}_{\theta}}(x)=\left(x_{0}+\tan \theta\left\|x_{r}\right\|\right) u_{x}^{2}, \\
& \Pi_{\mathscr{K}_{\theta}}(x+h)=\left(x_{0}+h_{0}+\tan \theta\left\|x_{r}+h_{r}\right\|\right) u_{x+h}^{2} \\
& \Pi_{\mathscr{K}_{\theta}}^{\prime}(x ; h)=\frac{1}{\tan \theta+\cot \theta} \times \\
& {\left[\begin{array}{cc}
\cot \theta & \widetilde{x}_{r}^{\mathrm{T}} \\
\tilde{x}_{r} & \frac{x_{0}+\left\|x_{r}\right\| \tan \theta}{\left\|x_{r}\right\|} I-\frac{x_{0}}{\left\|x_{r}\right\|} \widetilde{x}_{r} \widetilde{x}_{r}^{\mathrm{T}}
\end{array}\right]\left[\begin{array}{c}
h_{0} \\
h_{r}
\end{array}\right] .}
\end{aligned}
$$

By directly computations, we get

$$
\begin{aligned}
& \left\|x_{r}+h_{r}\right\|=\left\|x_{r}\right\|+\left\langle\widetilde{x}_{r}, h_{r}\right\rangle+O\left(\left\|h_{r}\right\|^{2}\right), \\
& \frac{x_{r}+h_{r}}{\left\|x_{r}+h_{r}\right\|}=\frac{x_{r}}{\left\|x_{r}\right\|} \\
& \quad+\frac{1}{\left\|x_{r}\right\|}\left(I-\widetilde{x}_{r} \widetilde{x}_{r}^{\mathrm{T}}\right) h_{r}+O\left(\left\|h_{r}\right\|^{2}\right),
\end{aligned}
$$

and

$$
\begin{aligned}
& \Pi_{\mathscr{K}_{\theta}}^{\prime}(x ; h)= \\
& {\left[\begin{array}{c}
\frac{1}{1+\tan ^{2} \theta}\left(h_{0}+\tan \theta\left\langle\widetilde{x}_{r}, h_{r}\right\rangle\right) \\
\frac{1}{\cot \theta+\tan \theta}\left(h_{0} \widetilde{x}_{r}+\frac{x_{0}+\left\|x_{r}\right\| \tan \theta}{\left\|x_{r}\right\|} h_{r}\right)+\mathbf{B}
\end{array}\right]} \\
& \mathbf{B}=-\frac{x_{0}}{\left\|x_{r}\right\|}\left\langle\widetilde{x}_{r}, h_{r}\right\rangle \widetilde{x}_{r} .
\end{aligned}
$$
By letting $\quad \widetilde{\Pi}^{h}:=$
$\left(1+\tan ^{2} \theta\right)\left(\Pi_{\mathscr{K}_{\theta}}(x+h)-\Pi_{\mathscr{K}_{\theta}}(x)-\Pi_{\mathscr{K}_{\theta}}^{\prime}(x ; h)\right)$,
then one has

$$
\begin{gathered}
\widetilde{\Pi}_{0}^{h}=O\left(\left\|h_{r}\right\|^{2}\right)=O\left(\|h\|^{2}\right), \\
O\left(\left\|h_{r}\right\|^{2}\right)-\left(x_{0}+\tan \theta\left\|x_{r}\right\| \widetilde{x}_{r} \tan \theta\right) \\
-h_{0} \tan \theta \widetilde{x}_{r}-\frac{x_{0} \tan \theta+\left\|x_{r}\right\| \tan ^{2} \theta}{\left\|x_{r}\right\|} h_{r} \\
+\frac{x_{0} \tan \theta}{\left\|x_{r}\right\|}\left\langle\widetilde{x}_{r}, h_{r}\right\rangle \widetilde{x}_{r} \\
=h_{0}\left\langle\widetilde{x}_{r}, h_{r}\right\rangle+O\left(\left\|h_{r}\right\|^{2}\right)=O\left(\|h\|^{2}\right),
\end{gathered}
$$

where the last equation holds by the fact that

$$
\begin{aligned}
& \frac{\left\|h_{0}\left\langle\widetilde{x}_{r}, h_{r}\right\rangle\right\|}{\|h\|^{2}} \leq \frac{\left|h_{0}\right| \cdot|| x_{r}\|\cdot\| h_{r} \|}{\|h\|^{2}} \\
& \leq \frac{\left\|x_{r}\right\| \cdot\|h\|^{2}}{2\|h\|^{2}}=\frac{\left\|x_{r}\right\|}{2} .
\end{aligned}
$$

Thus, (3.1) is fulfilled.

\section{APPLICATION}

In this section, we first establish the formulation for the Fréchet normal cone to the circular cone complementarity set $\Gamma$.

Theorem 4.1 Let $\Gamma$ be defined as in (2.4) and $(\bar{x}, \bar{y}) \in$ $\Gamma$. Then, we get Figure 4

Proof. We consider the following cases.

Case 1: $\mathrm{x}=0$ and $y \in$ int $\mathscr{K}_{\theta}^{*}$. Then, we get $\mathrm{x}-\mathrm{y}=-\mathrm{y}$, which implies that

$$
\begin{aligned}
& \lambda_{1}(x-y)=-y_{0}-\left\|y_{r}\right\| \cot \theta<0, \\
& \lambda_{2}(x-y)=-y_{0}+\left\|y_{r}\right\| \tan \theta .
\end{aligned}
$$

Since $y \in \operatorname{int} \mathscr{K}_{\theta}^{*}$, we get $y_{0} \tan \left(\frac{\pi}{2}-\theta\right)>$ $\left\|y_{r}\right\|$, i.e., $y_{0}>\left\|y_{r}\right\| \tan \theta$. Consequently, one has

$$
\lambda_{2}(x-y)=-y_{0}+\left\|y_{r}\right\| \tan \theta<0 .
$$

It follows from [ ${ }^{22}$, Lemma 3.1(a) and (3.6)] that

$$
\partial_{B}\left(\Pi_{\mathscr{K}_{\theta}}\right)(x-y)=\left\{\nabla \Pi_{\mathscr{K}_{\theta}}(x-y)\right\}=0 .
$$

By [22, Theorem 3.5(a)], we have

$$
\widehat{D}^{*} \Pi_{\mathscr{K}_{\theta}}(x-y)\left(y^{*}\right)=\left\{\nabla \Pi_{\mathscr{K}_{\theta}}(x-y) y^{*}\right\}=0 .
$$

Consequently, from (2.5), one obtains

$$
\widehat{N}_{\Gamma}(x, y)=\left\{(u, v) \mid u \in \mathbb{R}^{m+1}, v=0\right\} .
$$

Case 2: $x \in$ int $\mathscr{K}_{\theta}$ and $\mathrm{y}=0$. Then we get $\mathrm{x}-\mathrm{y}=\mathrm{x}$, so

$$
\begin{aligned}
& \lambda_{1}(x-y)=x_{0}-\left\|x_{r}\right\| \cot \theta<0 \\
& \lambda_{2}(x-y)=x_{0}+\left\|x_{r}\right\| \tan \theta
\end{aligned}
$$

It follows from [ ${ }^{22}$, Lemma 3.1(a), (3.6) and Theorem 3.5(a)] that

$$
\begin{aligned}
& \partial_{B}\left(\Pi_{\mathscr{K}_{\theta}}(x-y)=\left\{\nabla \Pi_{\mathscr{K}_{\theta}}(x-y)\right\}=I,\right. \\
& \widehat{D}^{*} \Pi_{\mathscr{K}_{\theta}}(x-y)\left(y^{*}\right)=\left\{\nabla \Pi_{\mathscr{K}_{\theta}}(x-y) y^{*}\right\}=y^{*} .
\end{aligned}
$$

Therefore, by (2.5), we have

$$
\widehat{N}_{\Gamma}(x, y)=\left\{(u, v) \mid u \in \mathbb{R}^{m+1}, v \in \mathbb{R}^{m+1}\right\} .
$$

Case 3: $x \in$ bd $\mathscr{K}_{\theta} \backslash\{0\}, y \in$ bd $\mathscr{K}_{\theta}^{*} \backslash\{0\}$ and $y^{\top} x=$ 0 . We get from Lemma 2.2 that $y=k\left(x_{0} \tan ^{2} \theta,-x_{r}\right)$ with $k=\frac{y_{0}}{x_{0}} \cot ^{2} \theta>0$, which implies that

$$
\begin{array}{r}
x-y=\left(x_{0}, x_{r}\right)-k\left(x_{0} \tan ^{2} \theta,-x_{r}\right) \\
=\left(\left(1-k \tan ^{2} \theta\right) x_{0},(1+k) x_{r}\right) .
\end{array}
$$

Moreover, we have

$$
\begin{aligned}
& \lambda_{1}(x-y) \\
& =\left(1-k \tan ^{2} \theta\right) x_{0}-(1+k)\left\|x_{r}\right\| \cot \theta \\
& =-k\left(1-\tan ^{2} \theta\right) x_{0}<0
\end{aligned}
$$




$$
\widehat{N}_{\Gamma}(x, y)= \begin{cases}\left\{(u, v) \mid u \in \mathbb{R}^{m+1}, v=0\right\} & \text { if } x=0, y \in \operatorname{int} \mathcal{K}_{\theta}^{\star} ; \\ \left\{(u, v) \mid u=0, v \in \mathbb{R}^{m+1}\right\} & \text { if } x \in \operatorname{int} \mathcal{K}_{\theta}, y=0 ; \\ \{(u, v) \mid u \perp x, v \perp y \text { and } & \text { if } x \in \text { bd } \mathcal{K}_{\theta} \backslash\{0\} \\ \left.x_{0} u+y_{0}\left(v_{0}^{*},-\cot ^{2} \theta v_{r}^{*}\right) \in \mathbb{R}\left(x_{0} \tan ^{2} \theta,-x_{r}\right)\right\} & \text { and } y \in \operatorname{bd} \mathcal{K}_{\theta}^{\star} \backslash\{0\} ; \\ \left\{(u, v) \mid u \in\left(y_{0} \cot ^{2} \theta,-y_{r}\right)^{\circ}, v \in \mathbb{R}_{-}\left(y_{0} \cot ^{2} \theta,-y_{r}\right)\right\} & \text { if } x=0, y \in b d \mathcal{K}_{\theta}^{\star} \backslash\{0\} ; \\ \left\{(u, v) \mid v \in\left(x_{0} \tan ^{2} \theta,-x_{r}\right)^{\circ}, u \in \mathbb{R}_{-}\left(x_{0} \tan ^{2} \theta,-x_{r}\right)\right\} & \text { if } x \in \text { bd } \mathcal{K}_{\theta} \backslash\{0\}, y=0 ; \\ \left\{(u, v) \mid u \in-\mathcal{K}_{\theta}^{\star}, v \in-\mathcal{K}_{\theta}\right\} & \text { if } x=0, y=0 .\end{cases}
$$

Figure 4: Theorem 4.1

and

$$
\begin{aligned}
& \lambda_{2}(x-y) \\
& =\left(1-k \tan ^{2} \theta\right) x_{0}+(1+k)\left\|x_{r}\right\| \cot \theta \\
& =\left(1-\tan ^{2} \theta\right) x_{0}>0 .
\end{aligned}
$$

By (3.6) in ${ }^{22}$, we get

$$
\begin{aligned}
& \nabla \Pi_{\mathscr{K}_{\theta}}(x-y)=\frac{1}{\tan \theta+\cot \theta} \times \\
& {\left[\begin{array}{cc}
\cot \theta & \widetilde{x}_{r}^{\mathrm{T}} \\
\widetilde{x}_{r} & \frac{1+\tan ^{2} \theta}{\tan \theta(1+k)} I-\frac{1-k \tan ^{2} \theta}{(1+k) \tan \theta} \widetilde{x}_{r} \widetilde{x}_{r}^{\mathrm{T}}
\end{array}\right] .}
\end{aligned}
$$

On the other hand, it follows from [ ${ }^{22}$, Theorem 3.5] and (2.5) that

$$
\begin{aligned}
& \widehat{N}_{\Gamma}(x, y) \\
& =\left\{(u, v) \mid-v \in \widehat{\mathrm{D}}^{*} \Pi_{\mathscr{K}_{\theta}}(x-y)(-u-v)\right\} \\
& =\left\{(u, v) \mid-v \in \nabla \Pi_{\mathscr{K}_{\theta}}(x-y)(-u-v)\right\} .
\end{aligned}
$$

Let $(u, v) \in \widehat{N}_{\Gamma}(x, y)$ and $x^{\prime} \in$ bd $\mathscr{K}_{\theta} \backslash\{0\}, x^{\prime} \in$ bd $\mathscr{K}_{\theta}^{*} \backslash\{0\}$ with $y^{\prime}=k\left(x_{0}^{\prime} \tan ^{2} \theta,-x_{r}^{\prime}\right)$. Then $y^{\prime} T x^{\prime}=$ 0 , which implies that $\left(\mathrm{x}^{\prime}, \mathrm{y}^{\prime}\right) \in \Gamma$. Consequently, one has

$$
\begin{aligned}
& \frac{\left\langle(u, v),\left(x^{\prime}, y^{\prime}\right)-(x, y)\right\rangle}{\left\|\left(x^{\prime}, y^{\prime}\right)-(x, y)\right\|}=\frac{\left\langle u, x^{\prime}-x\right\rangle+\left\langle v, y^{\prime}-y\right\rangle}{\left\|\left(x^{\prime}-x, y^{\prime}-y\right)\right\|} \\
& =\frac{\left\langle u, x^{\prime}-x\right\rangle+\left\langle k v,\left(x_{0}^{\prime} \tan ^{2} \theta,-x_{r}^{\prime}\right)-\left(x_{0} \tan ^{2} \theta,-x_{r}\right)\right\rangle}{\left\|\left(x^{\prime}-x, k\left(x_{0}^{\prime} \tan ^{2} \theta,-x_{r}\right)-k\left(\tan ^{2} \theta,-x_{r}\right)\right)\right\|} \\
& \geq \frac{\left\langle u+k\left(v_{0}^{*} \tan ^{2} \theta,-v_{r}^{*}\right), x^{\prime}-x\right\rangle}{\sqrt{1+k^{2}}\left\|x^{\prime}-x\right\|} .
\end{aligned}
$$

Since $(u, v) \in \widehat{N}_{\Gamma}(x, y)$, passing to the limit in (4.5), we get

$$
\begin{aligned}
& \limsup \\
& \limsup _{\left(x^{\prime}, y^{\prime}\right) \stackrel{\text { bd } \mathscr{K}_{\theta} \backslash\{0\}}{\longrightarrow}(x, y)} \frac{\frac{\left\langle u+k\left(v_{0}^{*} \tan ^{2} \theta,-v_{r}^{*}\right), x^{\prime}-x\right\rangle}{\left\|x^{\prime}-x\right\|} \leq}{\left\|\left(x^{\prime}, y^{\prime}\right)-(x, y)\right\|} \leq
\end{aligned}
$$

which implies that

$$
u+k\left(v_{0}^{*} \tan ^{2} \theta,-v_{r}^{*}\right) \in \widehat{N}_{\text {bd } \mathscr{K}_{\theta} \backslash\{0\}}(x)
$$

By $x \in$ bd $\mathscr{K}_{\theta} \backslash\{0\}$, there exists $r>0$ such that $0 \notin B(x, r)$ and $u_{0} \neq 0, u_{r} \neq 0$ for all $u \in B(x, r)$. For each $x \in$
$\mathbb{R}^{m+1} \backslash\{0\}$, we put $\varphi(x)=x_{0} \tan \theta-\left\|x_{r}\right\|$ if $x \neq 0$ and $\varphi(0)=1$. Then, bd $\mathscr{K}_{\theta} \backslash\{0\}$ can be expressed by

$$
\text { bd } \mathscr{K}_{\theta} \backslash\{0\}=\{x \mid \varphi(x)=0\}=\varphi^{-1}(0) .
$$

Moreover, we can check that $\varphi$ is continuously differentiable on $B(x, r)$, so $\nabla \varphi(u)$ has the full rank whenever $u \in B(x, r)$. It follows from [ ${ }^{27}$, Example 6.8] that

$$
\begin{array}{r}
\widehat{N}_{\text {bd } \mathscr{K}_{\theta} \backslash\{0\}}(x)=\left\{\nabla \varphi(x)^{*} u \mid u \in \mathbb{R}\right\} \\
=\mathbb{R}\left(x_{0} \tan ^{2} \theta,-x_{r}\right) .
\end{array}
$$

Taking (4.6) with $k=\frac{y_{0}}{x_{0}}$ into account, we have

$$
\begin{aligned}
& u+\frac{y_{0}}{x_{0}} \cot ^{2} \theta\left(v_{0}^{*} \tan ^{2} \theta,-v_{r}^{*}\right) \\
& \quad \in \mathbb{R}\left(x_{0} \tan ^{2} \theta,-x_{r}\right) .
\end{aligned}
$$

On the other hand, for each $n \in N$, let $x_{n}:=x, y_{n}:=$ $\left(1+\frac{1}{n}\right) y=\left(1+\frac{1}{n}\right) k\left(x_{0} \tan ^{2} \theta,-x_{r}\right)$, then $\left(x_{n}, y_{n}\right) \in \Gamma$ for all $n \in N$. Similarly to (4.5), one gets

$$
\frac{\left\langle(u, v),\left(x_{n}, y_{n}\right)-(x, y)\right\rangle}{\left\|\left(x_{n}, y_{n}\right)-(x, y)\right\|}=\frac{\left\langle v, \frac{1}{n} y\right\rangle}{\frac{1}{n}\|y\|}
$$

Passing to limsup, it follows from the definition of regular normal cone that $\langle v, y\rangle \leq 0$. Otherwise, if we take $x_{n}^{\prime}:=x, y_{n}^{\prime}=\left(1-\frac{1}{n}\right) y$ for each $n \in N$ then by the similar method, we obtain $\langle v, y\rangle \geq 0$. Therefore, $\langle v, y\rangle=0$, i.e., $\mathrm{v} \perp$ y. Similarly, one gets $\mathrm{u} \perp \mathrm{x}$. Consequently,

$\widehat{N}_{\Gamma}(x, y) \subset\{(u, v) \mid u \perp x, v \perp y$ and $\left.x_{0} u+y_{0}\left(v_{0}^{*},-\cot ^{2} \theta v_{r}^{*}\right) \in \mathbb{R}\left(x_{0} \tan ^{2} \theta,-x_{r}\right)\right\}$.

For the inverse of the above inclusion, let $(u, v)$ be satisfied $u \perp x, v \perp y$ and $x_{0} u+y_{0}\left(v_{0}^{*},-\cot \theta v_{r}^{*}\right)=$ $\alpha\left(x_{0} \tan ^{2} \theta,-x_{r}\right)$ with some $\alpha \in R$. We need to prove that $-v \in \nabla \Pi_{\mathscr{K}_{\theta}}(x-y)(-u-v)$ with $\nabla \Pi(x)$ as in (4.3). Indeed, one has

$$
\begin{aligned}
& \cot \theta\left(-u_{0}-v_{0}\right)+\left\langle\widetilde{x}_{r},-u_{r}-v_{r}\right\rangle \\
& =\cot \theta\left(-u_{0}-v_{0}\right)+\frac{u_{0} x_{0}-v_{0} x_{0} \tan ^{2} \theta}{x_{0} \tan \theta} \\
& =-(\cot \theta+\tan \theta) v_{0} .
\end{aligned}
$$




$$
\begin{aligned}
& A:=\tilde{x}_{r}\left(-u_{0}-v_{0}\right)+\frac{1+\tan ^{2} \theta}{\tan \theta(1+k)}\left(-u_{r}-v_{r}\right)-\frac{1-k \tan ^{2} \theta}{(1+k) \tan \theta}\left\langle\tilde{x}_{r},-u_{r}-v_{r}\right) \tilde{x}_{r} \\
& =\tilde{x}_{r}\left(-u_{0}-v_{0}\right)+\frac{1-k \tan ^{2} \theta}{(1+k) \tan \theta}\left(-\left(u_{0}+v_{0}\right) \cot \theta+v_{0}(\cot \theta+\tan \theta)\right) \tilde{x}_{r}+\alpha x_{r} \frac{\cot \theta+\tan \theta}{1+k}-(\cot \theta+\tan \theta) v_{r} \\
& =\left(-u_{0}-v_{0}+\frac{1-k \tan ^{2} \theta}{(1+k) \tan \theta}\left(-\left(u_{0}+v_{0}\right) \cot \theta+v_{0}(\cot \theta+\tan \theta)\right)+\alpha|| x_{r}|| \frac{\cot \theta+\tan \theta}{1+k}\right) \tilde{x}_{r}-(\cot \theta+\tan \theta) v_{r} .
\end{aligned}
$$

Figure 5: Equation 4.9

Moreover, since $u_{r}-k v_{r}=-\alpha x_{r}$, we get

$$
u_{r}+v_{r}=(1+k) v_{r}-\alpha x_{r} .
$$

It follows from (4.7) and (4.8), we have equation 4.9 in Figure 5

We next show that

$$
\begin{aligned}
& -u_{0}-v_{0}+\frac{1-k \tan ^{2} \theta}{(1+k) \tan \theta} \times \\
& \left(-\left(u_{0}+v_{0}\right) \cot \theta+v_{0}(\cot \theta+\tan \theta)\right) \\
& +\alpha\left\|x_{r}\right\| \frac{\cot \theta+\tan \theta}{1+k}=0 .
\end{aligned}
$$

By replacing $\left\|x_{r}\right\|=x_{0} \tan \theta$ and $\alpha x_{0} \tan ^{2} \theta=$ $u_{0} x_{0} 0+k v_{0} \tan ^{2} \theta$, the above equality is equivalent to

$$
\begin{aligned}
& u_{0}\left(-1+\frac{1+\tan ^{2} \theta}{(1+k) \tan ^{2} \theta}-\frac{1-k \tan ^{2} \theta}{(1+k) \tan ^{2} \theta}\right) \\
& +v_{0}\left(-1+k \frac{1+\tan ^{2} \theta}{1+k}+\frac{1-k \tan ^{2} \theta}{1+k}\right)=0,
\end{aligned}
$$

which is always fulfilled by the fact that

$$
\begin{aligned}
& -1+\frac{1+\tan ^{2} \theta}{(1+k) \tan ^{2} \theta}-\frac{1-k \tan ^{2} \theta}{(1+k) \tan ^{2} \theta}=0, \\
& -1+\frac{1+\tan ^{2} \theta}{1+k}-\frac{1-k \tan ^{2} \theta}{1+k}=0 .
\end{aligned}
$$

Taking (4.9) into account, one gets

$$
A=-v_{r}(\tan \theta+\cot \theta)
$$

It follows from (4.3), (4.7) and (4.10) that $\nabla \Pi_{\mathscr{K}_{\theta}}(x-$ $y)(-u-v)=-v$, so $(u, v) \in \widehat{N}_{\Gamma}(x, y)$. Hence, in this case, we get

$$
\begin{aligned}
& \widehat{N}_{\Gamma}(x, y)=\{(u, v) \mid u \perp x, v \perp y \text { and } \\
& \left.x_{0} u+y_{0}\left(v_{0}^{*},-\cot ^{2} \theta v_{r}^{*}\right) \in \mathbb{R}\left(x_{0} \tan ^{2} \theta,-x_{r}\right)\right\} .
\end{aligned}
$$

Case 4: $x=0, y \in$ bd $\mathscr{K}_{\theta}^{*} \backslash\{0\}$. In this case, we get $x-y=-y=\left(-y_{0},-y_{r}\right)$. Thus, one has $\lambda_{1}(x-y)=$ $-y_{0}-\left\|y_{r}\right\| \cot \theta<0$ and $\lambda_{2}(x-y)=-y_{0}+\left\|y_{r}\right\|$ $\tan \theta=0$.

By $\left[{ }^{22}\right.$, Theorem $\left.3.5(\mathrm{c})\right]$, we obtain

$$
\begin{aligned}
& \widehat{D}^{*} \Pi_{\mathscr{K}_{\theta}}(x-y)(-u-v)=\left\{w \in \mathbb{R}^{m+1} \mid\right. \\
& \left.w \in \mathbb{R}_{+} u_{x-y}^{2},\left\langle-u-v-w, u_{x-y}^{2}\right\rangle\right\} \geq 0,
\end{aligned}
$$

where $u_{x-y}^{2}=\frac{1}{1+\tan ^{2} \theta}\left[\begin{array}{cc}1 & 0 \\ 0 & \tan \theta\end{array}\right]\left[\begin{array}{c}1 \\ -\widetilde{y}\end{array}\right]$. It follows from (2.5) and (4.11) that $(u, v) \in \widehat{N}_{\Gamma}(x, y)$ if and only if there exists $\alpha \geq 0$ satisfying

$$
\begin{aligned}
& -v=\alpha \frac{1}{1+\tan ^{2} \theta}\left[\begin{array}{cc}
1 & 0 \\
0 & \tan \theta
\end{array}\right]\left[\begin{array}{c}
1 \\
-\widetilde{y}
\end{array}\right] \\
& =\frac{\alpha}{(\cot \theta+\tan \theta)\left\|y_{r}\right\|}\left[\begin{array}{c}
y_{0} \\
-y_{r}
\end{array}\right] \\
& =\frac{\alpha}{(\cot \theta+\tan \theta)\left\|y_{r}\right\|} \hat{y}
\end{aligned}
$$

and

$$
\begin{aligned}
& \frac{1}{1+\tan ^{2} \theta}\left[\begin{array}{cc}
1 & 0 \\
0 & \tan \theta
\end{array}\right]\left[\begin{array}{c}
1 \\
-\tilde{y}
\end{array}\right] u \\
& =\frac{1}{(\cot \theta+\tan \theta)\left\|y_{r}\right\|}\langle\hat{y}, u\rangle \leq 0 .
\end{aligned}
$$

This is equivalent to $v \in \mathbb{R} \_\widehat{y}$ and $u \in \widehat{y}^{\circ}$, which implies that

$$
\begin{aligned}
& \widehat{N}_{\Gamma}(x, y)=\left\{(u, v) \mid u \in\left(y_{0} \cot ^{2} \theta,-y_{r}\right)^{\circ},\right. \\
& \left.v \in \mathbb{R} \_\left(y_{0} \cot ^{2} \theta,-y_{r}\right)\right\} .
\end{aligned}
$$

Case 5: $x \in$ bd $\mathscr{K}_{\theta} \backslash\{0\}$ and $\mathrm{y}=0$. Similarly to Case 4, we get

$$
\begin{aligned}
& \widehat{N}_{\Gamma}(x, y)=\left\{(u, v) \mid v \in\left(x_{0} \tan ^{2} \theta,-x_{r}\right)^{\circ},\right. \\
& \left.u \in \mathbb{R}_{-}\left(x_{0} \tan ^{2} \theta,-x_{r}\right)\right\} .
\end{aligned}
$$

Case 6: $x=0, y=0$. Then, we have $\lambda_{1}(x-y)=\lambda_{2}(x-$ $y)=0$, and thus from [ ${ }^{22}$, Theorem $\left.3.5(\mathrm{~d})\right]$, one gets

$$
\begin{array}{r}
\widehat{D}^{*} \Pi_{\mathscr{K}_{\theta}}(x-y)(-u-v)=\left\{w \in \mathbb{R}^{m+1}\right. \\
\left.\mid w \in \mathscr{K}_{\theta},-u-v-w \in \mathscr{K}_{\theta}^{*}\right\} .
\end{array}
$$

It follows from $(2.5)$ that $(u, v) \in \widehat{N}_{\Gamma}(x, y)$ if and only if $-v \in \mathscr{K}_{\theta}$ and $-u \in \mathscr{K}_{\theta}^{*}$. Consequently, we obtain

$$
\widehat{N}_{\Gamma}(x, y)=\left\{(u, v) \mid u \in \mathscr{K}_{\theta}^{*}, v \in-\mathscr{K}_{\theta}\right\}
$$

In what follows, we present necessary conditions for the following mathematical program with circular cone complementarity constraints:

$$
\begin{array}{lc}
\operatorname{Min} f(x) & (\mathrm{MPCCC}) \\
\text { subject to } \mathscr{K}_{\theta} \ni G(x) \perp H(x) \in \mathscr{K}_{\theta}^{*}
\end{array}
$$

where $\theta_{i} \in\left(0, \frac{\pi}{2}\right)$ and $f: \mathbb{R}^{n} \rightarrow \mathbb{R}, G, H: \mathbb{R}^{n} \rightarrow \mathbb{R}^{m+1}$ are continuously differentiable and $\mathscr{K}_{\theta} \subset \mathbb{R}^{m+1}$ is a circular cone. The problem (MPCCC) is a generalization of the mathematical program with second-order 
cone complementarity constraints (MPSOCC) studied in ${ }^{10,11,13}$. The feasible set of (MPCCC) is defined by

$$
\Xi:=\{x \mid F(x) \in \Gamma\},
$$

where $\Gamma$ is given as (2.4) and $F(x)=(G(x), H(x))$.

Definition 4.2 Let $\bar{x}$ be a feasible solution of (MPCCC). We say that $\bar{x}$ is a local optimal solution of (MPCCC) if there exist there exists $\delta>0$ such that

$$
f(x) \geq f(\bar{x}) \quad \forall x \in B_{\delta}(\bar{x}) \cap \Xi .
$$

Theorem 4.3 Let $\bar{x}$ be a local optimal solution of (MPCCC) and let $\nabla \mathrm{F}(\bar{x})$ be surjective. Then

$$
0 \in \nabla f(\bar{x})+\nabla F(\bar{x})^{\mathrm{T}} \widehat{N}_{\Gamma}(F(\bar{x})) .
$$

Proof. It is easy to observe that $\bar{x}$ is a local optimal solution of (MPCCC) if $\bar{x}$ is a local optimal solution the function $f(x)+\delta_{\Xi}(x)$. We have from [ ${ }^{25}$, Proposition 1.10] that

$$
0 \in \widehat{\partial}\left(f+\delta_{\Xi}\right)(\bar{x}) .
$$

Using [ ${ }^{25}$, Corollary 1.12.1], we get $0 \in \nabla f(\bar{x})+\widehat{\partial} \delta_{\Xi}(\bar{x})$ which is equivalent to $0 \in \nabla f(\bar{x})+\widehat{N}_{\Xi}(\bar{x})$. Using Lemma 2.1, we obtain $0 \in \nabla f(\bar{x})+\nabla F(\bar{x})^{\mathrm{T}} \widehat{N}_{\Gamma}(F(\bar{x}))$.

Finally, we give an example to illustrate Theorem 4.3. Example 4.4 Consider the following problem

$$
\operatorname{Min} x_{1}^{2}+2 x_{2}^{2}
$$

subject to

$$
\begin{aligned}
& \mathscr{K}_{\pi / 4} \ni\left(\begin{array}{l}
x_{1} \\
x_{2}
\end{array}\right)=: G(x) \perp H(x) \\
& :=\left(\begin{array}{c}
x_{1} \\
-x_{2}
\end{array}\right) \in \mathscr{K}_{\pi / 4}^{*}
\end{aligned}
$$

It is easy to check that $\bar{x}:=\left(\begin{array}{l}0 \\ 0\end{array}\right)$ is a local solution of this problem. By direct computations, we get

$$
\begin{aligned}
& \qquad f(\bar{x})=(0,0) \text { and } \nabla \mathrm{F}(\overline{\mathrm{x}})=(\mathrm{I}, \widehat{\mathrm{I}}), \\
& \text { where } f(x)=x_{1}^{2}+2 x_{2}^{2}, \mathrm{~F}(\mathrm{x})=(\mathrm{G}(\mathrm{x}), \mathrm{H}(\mathrm{x})), \mathrm{I}= \\
& {\left[\begin{array}{rr}
1 & 0 \\
0 & 1
\end{array}\right] \text { and } \widehat{\mathrm{I}}=\left[\begin{array}{cc}
1 & 0 \\
0 & -1
\end{array}\right] . \text { Therefore, }} \\
& (0,0) \in \nabla f(\bar{x})+\nabla \mathrm{F}(\bar{x})^{\mathrm{T}} \widehat{N}_{\Gamma}(G(\bar{x}), H(\bar{x})) \\
& =(0,0)+\mathscr{K}_{\pi / 4} \times\left(-\mathscr{K}_{\pi / 4}\right) .
\end{aligned}
$$

\section{CONCLUSION}

In this paper, we have first shown the calmly Bdifferentiable property of the projector onto a circular cone. Then, we presented the exact formula for computing the Fréchet normal cones to the circular cone complementarity set. Finally, we have provided firstorder necessary conditions for local optimal solutions to mathematical programs with circular cone complementarity constraints.

For possible developments, we are planning to employ the obtained results in calculating the directionally limiting normal cone of the circular cone complementarity set. Moreover, inspired by ${ }^{13}$, sufficient conditions for the error bound property of circular cone complementarity problems would be established by using the current approach.

\section{COMPETING INTERESTS}

The author(s) declare that they have no competing interests.

\section{ACKNOWLEDGMENTS}

This work was partially supported by Dong Thap University and the Vietnam Ministry of Education and Training under the grant number B2019.SPD.01.

\section{REFERENCES}

1. Alizadeh F, Goldfarb D. Second-order cone programming Mathematical Programming. 2003;95:3-51. Available from: https://doi.org/10.1007/s10107-002-0339-5.

2. Bonnans JF, Ramirez $\mathrm{CH}$. Perturbation analysis of secondorder cone programming problems. Mathematical Programming. 2005;104:205-227. Available from: https://doi.org/10. 1007/s10107-005-0613-4.

3. Bonnans JF, Shapiro A. Perturbation analysis of optimization problems. Springer-Verlag, New York. 2000;Available from: https://doi.org/10.1007/978-1-4612-1394-9.

4. Hang NTV, Mordukhovich BS, Sarabi ME. Second-order variational analysis in second-order cone programming. Mathematical Programming. 2020;180:75-116. Available from: https://doi.org/10.1007/s10107-018-1345-6.

5. Liu YJ, Zhang LW. Convergence of the augmented Lagrangian method for nonlinear optimization problems over secondorder cones. Journal of Optimization Theory and Applications. 2008;139:557-575. Available from: https://doi.org/10. 1007/s10957-008-9390-6.

6. Mordukhovich BS, Outrata JV, Sarabi ME. Full stability of locally optimal solution in second-order cone programming. SIAM Journal of Optimization. 2014;24:1581-1613. Available from: https://doi.org/10.1137/130928637.

7. Outrata JV, Ramírez H. On the Aubin property of critical points to perturbed second-order cone programs. SIAM Journal of Optimization. 2011;21:798-823. Available from: https://doi. org/10.1137/100807168.

8. Lobo MS, Vandenberghe L, Boyd S, Lebret H. Applications of second-order cone programming. Linear Algebra and Its Applications. 1998;284:193-228. Available from: https://doi.org/ 10.1016/S0024-3795(98)10032-0. 
9. Hayashi S, Yamashita N, Fukushima M. A combined smoothing and regularized method for monotone second-order cone complementarity problems. SIAM Journal of Optimization. 2005;15:593-615. Available from: https://doi.org/10.1137/ S1052623403421516.

10. Liang YC, Zhu XD, Lin GH. Necessary optimality conditions for mathematical programs with second-order cone complementarity constraints. Set-Valued and Variational Analysis. 2014;22:59-78. Available from: https://doi.org/10.1007/ s11228-013-0250-7.

11. Ye JJ, Zhou J. Exact formulas for the proximal/regular/limiting normal cone of the second- order cone complementarity set. Mathematical Programming. 2017;162:33-50. Available from: https://doi.org/10.1007/s10107-016-1027-1.

12. Ye JJ, Zhou J. First-order optimality conditions for mathematical programs with second- order cone complementarity constraints. SIAM Journal of Optimization. 2016;26:2820-2846. Available from: https://doi.org/10.1137/16M1055554.

13. Ye JJ, Zhou J. Verifiable sufficient conditions for the error bound property of second-order cone complementarity problems. Mathematical Programming. 2018;171:361-395. Available from: https://doi.org/10.1007/s10107-017-1193-9.

14. Chang $Y L$, Yang $C Y$, Chen JS. Smooth and nonsmooth analyses of vector-valued functions associated with circular cones. Nonlinear Analysis: Theory, Methods and Applications. 2013;85:160-173. Available from: https://doi.org/10.1016/j.na. 2013.01.017.

15. Glineur F, Terlaky T. Conic formulation for Ip-norm optimization. Journal of Optimization Theory and Applications. 2004;122:285-307. Available from: https://doi.org/10.1023/B: JOTA.0000042522.65261.51

16. Thinh VD, Chuong TD. Subdifferentials and derivatives with respect to a set and applications to optimization. Applicable Analysis. 2019;98:1005-1026. Available from: https://doi.org/ 10.1080/00036811.2017.1408081

17. Thinh VD, Chuong TD, Anh NLH. Optimality Conditions for Circular Cone Complementarity Programs. Optimization. 2020;Available from: https://doi.org/10.1080/02331934.2020.
1808645.

18. Wang $G Q$, Bai $Y Q$. A class of polynomial interior point algorithms for the Cartesian P-matrix linear complementarity problem over symmetric cones. Journal of Mathematica Analysis and Applications. 2012;152:739-772. Available from: https://doi.org/10.1007/s10957-011-9938-8.

19. Zhou J, Chang YL, Chen JS. The H-differentiability and calmness of the circular cone functions. Journal of Global Optimization. 2015;63:811-833. Available from: https://doi.org/10 1007/s10898-015-0312-5.

20. Zhou J, Chen JS. Properties of circular cone and spectral factorization associated with circular cone. Journal of Nonlinear Convex Analysis. 2013;14:807-816. Available from: https: //doi.org/10.1186/1029-242X-2013-571.

21. Zhou J, Chen JS. Monotonicity and circular cone monotonicity associated with circular cones. Set-Valued and Variational Analysis. 2017;25:211-232. Available from: https://doi.org/10. 1007/s11228-016-0374-7.

22. Zhou J, Chen JS, Mordukhovich BS. Variational analysis of circular cone programs. Optimization. 2015;64:113-147. Available from: https://doi.org/10.1080/02331934.2014.951043.

23. Zhou J, Tang J, Chen JS. Parabolic second-order directional differentiability in the Hadamard sense of the vector-valued Functions associated with circular cones. Journal of Optimization Theory and Applications. 2017;172:802-823. Available from: https://doi.org/10.1007/s10957-016-0935-9.

24. Mordukhovich BS. Variational analysis and generalized differentiation I. Springer, Berlin. 2006;Available from: https: //doi.org/10.1007/3-540-31247-1.

25. Kruger AY. On Fréchet subdifferentials. Journal of Mathematical Sciences. 2003;116:3325-3358. Available from: https: //doi.org/10.1023/A:1023673105317.

26. Combettes BL, Reyes NN. Moreau's decomposition in Banach spaces. Mathematical Programming. 2013;139:103-114 Available from: https://doi.org/10.1007/s10107-013-0663-y.

27. Rockafellar RT, Wets RJB. Variational Analysis. Springer, Berlin. 1998;Available from: https://doi.org/10.1007/978-3 642-02431-3. 